\title{
RELACIÓN TERAPÉUTICA: EL PILAR DE LA PROFESIÓN ENFERMERA
}

\author{
THERAPEUTIC RELATIONSHIP: THE PILLAR OF THE NURSING PROFESSION
}

${ }^{*}$ Vidal Blan, $\mathrm{R},{ }^{* *}$ Adamuz Tomás, J, *Feliu Baute, $\mathrm{P}$.

*Enfermera. Servicio de Neonatología. Hospital de Sant Joan de Déu. **Enfermero becario de investigación. Servicio de Enfermedades Infecciosas. Hospital Universitari de Bellvitge. Barcelona

Palabras clave: Relación terapéutica AND enfermería, Relación de ayuda AND enfermería; Alianza terapéutica AND enfermería

Keywords: Therapeutic Relationship and Nursing, Aid Relationship and Nursing, and Therapeutic Alliance and Nursing.

\section{RESUMEN}

En esta revisión se presenta una visión global de la relación terapéutica y su relevancia en la profesión enfermera. A través de ésta, se quiere demostrar la importancia de la adquisición de habilidades y actitudes fundamentales a la hora de iniciar una relación de ayuda. Una de las características de la enfermería es el concepto del cuidado a través del cual damos significado a la profesión. Entre las necesidades de los cuidados encontramos la más importante de ellas que es la necesidad de establecer una correcta relación terapéutica.

Si los estudiantes de enfermería están entrenados en estas habilidades, conseguiremos profesionales mejores preparados, que contemplarán a las personas en su globalidad, proporcionando una atención integral. Ésta es una demanda de la sociedad actual, la cual conllevaría a una mejora de la salud individual.

\section{ABSTRACT}

This revision presents a global vision about the therapeutic relationship and its importance for nursing professionals. With this, we will show the importance of the acquisition of skills and attitudes for initiating the therapeutic relationship. One of the most important characteristic of nursing is the concept of care which is the essence of this profession. Among the most important needs, we found the need to establish a correct therapeutic relationship.

If nursing students are coached in these skills, we will be able to achieve better prepared professionals, who have a holistic vision of the patient, they offering an integral attention to the patient. This is a demand to our current society, which assures the improvement of individual health. 


\section{INTRODUCCION}

En esta revisión se presenta una visión global de la relación terapéutica y su relevancia en la profesión enfermera. Es de gran importancia la adquisición de habilidades y actitudes fundamentales a la hora de iniciar una relación de ayuda (1). Por ello queremos demostrar a los docentes de esta disciplina, profesionales de enfermería y al resto de profesionales sanitarios, la importancia de formar al colectivo enfermero en este aspecto. En la práctica profesional la relación persona - enfermera aparece como una constante y es que las enfermeras teóricas definen la profesión como una relación de ayuda (2). Se puede afirmar que la relación terapéutica es una relación profesional (3) basada en un proceso de interacción e influencia social cuyo objetivo es la curación del enfermo (4).

La relación terapéutica se podría definir como una negociación intersubjetiva en la que a través del discurso que se establece entre terapeuta y paciente se va construyendoreconstruyendo el binomio salud-enfermedad (5). Por lo tanto es considerada como un proceso, método o instrumento, por medio del cual son transmitidos los significados entre personas y grupos (6).

En el ámbito de los servicios sanitarios, muchos problemas se originan cuando la comunicación es mala. Uno de ellos es la falta de comunicación con los profesionales sanitarios, que es la causa más importante de insatisfacción de los pacientes hospitalizados. Entre un 30 y un $50 \%$ de los pacientes no muestran adhesión al tratamiento a consecuencia de una deficiente comunicación con el personal. La falta de información sobre los procesos de enfermedad provoca un retraso en la búsqueda de tratamiento y un agravamiento de dichos procesos. La deficiente comunicación profesional-usuario suele dar lugar a que este último olvide las instrucciones del primero, perjudicando a su recuperación. Por último, está demostrado que el establecimiento de una adecuada interacción entre el profesional y los usuarios de los servicios sanitarios, garantiza la adopción de medidas y estrategias preventivas por parte del usuario.

Teniendo en cuenta estos problemas, se debe evitar caer en el tópico de que el saber comunicarse es un arte más que una técnica no susceptible de enmarcarse dentro de un proceso de enseñanza-aprendizaje (4). Actualmente las escuelas universitarias no contemplan como enseñanza obligatoria el aprendizaje de las habilidades sociales para establecer una relación terapéutica. Si no se tiene una idea clara de lo que es la comunicación y la relación terapéutica podemos caer en el famoso y cada vez más real síndrome del burn-out (7), al obviar una de las habilidades más importantes de la profesión enfermera: la comunicación interpersonal.

En todo proceso comunicativo influyen unos factores personales tanto por parte del emisor como del receptor, cada uno de los cuales aporta sus propios valores, cualidades y biografía personal. Hay que tener en cuenta que la enfermera no sólo tiene una historia personal, sino que también pertenece a un sistema cultural en el que ha sido socializada. Los principales elementos de los factores personales son: la orientación teórica, que se refiere al enfoque particular que tiene el observador, el uso del lenguaje, las variables socioculturales, como la edad, género y étnica y las variables individuales, que son las características de la personalidad manifestadas a través de la conducta y la apariencia (5).

A partir de esto iniciamos el "viaje" hacia el conocimiento y aplicación de la relación terapéutica en la profesión enfermera. 


\section{OBJETIVO}

Realizar una revisión bibliográfica basada en:

- Definir la relación terapéutica y sus características.

- Remarcar la importancia de la relación terapéutica en la profesión enfermera.

- Identificar su aplicación tanto en el ámbito académico como a nivel asistencial.

\section{METODOLOGÍA}

Para la elaboración de ésta revisión bibliográfica se ha realizado una búsqueda exhaustiva en las siguientes bases de datos:

- CINALH

- Medline

- Cuidatge

- Cuiden

La ecuación de búsqueda utilizada está basada en los siguientes conceptos:

- Relación terapéutica AND enfermería

- Relación de ayuda AND enfermería

- Alianza terapéutica AND enfermería

- Therapeutic relationship AND nursing

Los criterios de inclusión son:

- $\quad$ Artículos publicados a partir del año 2000

- Artículos relacionados con la profesión enfermera

Se han revisado 60 artículos, de los que se han seleccionado 21 por cumplir los criterios de inclusión y por su relevancia para los objetivos de la revisión.

\section{RESULTADOS}

\section{a) Comunicación verbal y no verbal}

Toda persona alguna vez a lo largo de su vida necesitará una asistencia sanitaria. En ese momento estaremos ante una persona que está viviendo una situación de temor, miedo o inseguridad, y necesitará ser comprendida para poder satisfacer sus necesidades. Los profesionales sanitarios no pueden solucionar este tipo de problemas de forma técnica, ya que deben tener una visión holística para contemplar la globalidad del problema. Para conseguir esto debemos establecer una comunicación efectiva con el paciente que nos permitirá establecer una relación terapéutica con este último, basada en un intercambio entre profesional sanitario y cliente. Esta relación interpersonal permitirá afrontar los problemas que aparezcan de manera conjunta, se puede denominar con diversos nombres: relación terapéutica, alianza terapéutica, relación de ayuda... Todos ellos con un mismo objetivo: ayudar al individuo, familia y comunidad a prevenir o actuar frente a la enfermedad (1). 
Esta comunicación designa el intercambio de mensajes, y es considerada como un proceso, método o instrumento, por medio del cual son transmitidos los significados entre personas y grupos. La personalización del diálogo que se ha de llevar a cabo no se refiere únicamente a la comunicación verbal, sino que contempla también los aspectos no verbales como: la escucha activa, la actitud empática, la aceptación incondicional (no hacer juicios de valor) y la asertividad. De esta manera para que la comunicación sea efectiva es necesario comprender al ser humano como ser activo y al cuidado como finalidad (6).

\section{b) La relación terapéutica como esencia de la profesión enfermera basada en la relación con el paciente.}

Peplau (1989) define enfermería como aquella profesión basada en la relación entre profesional y el paciente (persona individual) (8). Una de las características de la enfermería es el concepto del cuidado a través del cual damos significado a la profesión. Entre las necesidades de los cuidados encontramos la más importante de ellas que es la necesidad de establecer una correcta relación terapéutica. La ciencia enfermera es una ciencia guiada por la práctica y por las nuevas necesidades de cuidados, siendo una de ellas establecer un mayor cuidado del paciente basado en una relación terapéutica (9). Tal como dice el Colegio de Enfermería de Ontario, la profesión enfermera está centrada en la relación terapéutica, que se basa en el conocimiento y entrenamiento de habilidades para conseguir el bienestar del paciente (10).

\section{c) El poder terapéutico de las palabras en enfermería.}

Tal como describen los estudios presentados por Stickley y the College of Nurse of Ontario, establecer una efectiva relación terapéutica contribuye a una disminución del tiempo de recuperación. El primero de los artículos presenta como resultados que el aumento de la calidad de la comunicación enfermera disminuye la ansiedad, mejora sus habilidades de autocuras y se consigue una mayor adherencia al tratamiento. Además los pacientes están más insatisfechos con una pobre comunicación que con otros aspectos de las curas durante la estancia hospitalaria. Actualmente muchas personas tienen enfermedades psicosociales que podrían ser tratadas a partir de una relación terapéutica, cosa que disminuiría el abuso de antidepresivos u otro tipo de tratamientos (11).

El segundo artículo afirma que una buena relación de ayuda contribuye a la salud del paciente y a su bienestar (10).

En relación con este punto, en el III Congreso Nacional de la Sociedad Española de Psicooncología, se afirmó que la sanidad tiene que orientarse no hacia la enfermedad, sino hacia el enfermo. Josune Onaindia (jefa de hospitalización) añade que lo importante es que en la asistencia sanitaria haya pacientes, no enfermedades ni número de camas. Txaro Beobide (representante de los pacientes) refiere que la necesidad de ayuda es diferente en cada caso, por lo que el equipo asistencial realizará una adecuada evaluación de cada paciente, y de su situación socio-familiar. Este artículo afirma que las particularidades personales tanto de cada enfermo como de los profesionales que intervienen, influyen en la propia vivencia de la enfermedad (12).

\section{d) Importancia de la escucha activa y del dejar hablar.}

La alianza terapéutica entre enfermera y el paciente es una relación de dos personas extrañas entre las que se forma un "apego" especial, que lleva a una "transferencia" inconsciente, que hace que las palabras de la enfermera sean de gran importancia para el 
paciente (3). Contrariamente a lo que podríamos pensar, este vínculo no fomenta la dependencia sino todo lo contrario, pues lo que se intenta es hacer tomar conciencia al paciente de las armas que tiene para poder afrontar su proceso de enfermedad (7). También gracias a esta transferencia el paciente dice y cuenta cosas que no le diría a otra persona, y esto puede curarle. Para que esto ocurra la enfermera debe tener una actitud de escucha activa y de dejar hablar (3).

Es importante tener en cuenta que escuchar no es una acción pasiva, sino que es un arte o habilidad que sólo se puede alcanzar con la práctica. Hemos de incorporarla para poder desarrollar una relación terapéutica efectiva (11). La comunicación terapéutica que se utiliza no es igual que la comunicación social, ya que en la terapéutica tenemos la responsabilidad de la calidad de la comunicación con el paciente, pensando y escuchando con una metodología específica desde el rol de enfermería. En la comunicación terapéutica el paciente es el protagonista, el que habla, y el profesional el que escucha y lo acompaña en su proceso de elaboración.

Por lo tanto es muy importante que el paciente se sienta escuchado, sea escuchado y se escuche a sí mismo con la ayuda del profesional. Muchas veces el profesional de enfermería se queja de no disponer de tiempo para desarrollar esta actividad, pero puede que el paciente sólo necesite tres minutos, y esos tres minutos que se le dediquen hay que escucharlo con tranquilidad, interés, atención, respeto, sin interrumpirlo ni juzgarlo y dirigiéndose a él de manera empática y con respeto (13)

\section{e) Ingredientes para la consecución de una buena relación terapéutica.}

Dependiendo de la actitud que tengamos hacía el paciente realizamos un estilo diferente de relación de ayuda y por ello obtendremos diferentes resultados. Si nos centramos en el problema del paciente, en resolverlo de forma directa, y no se buscan los recursos propios del paciente sino que utilizamos los propios, se trata de un modelo autoritario. Si centramos el problema en el paciente y se adopta una actitud facilitadora y se implica al paciente para buscar la manera de resolver el problema, se trata de un modelo democrático o cooperativo. Por otro lado, si centramos el problema en la persona, en cómo vive el problema y adoptamos una actitud directiva y tomamos la responsabilidad de la situación del paciente y le sobreprotegemos, se trata de un estilo paternalista. Por último y para que se vea la diferencia, el modelo empático que es el ideal a seguir: se dirige a la persona, se adopta una actitud facilitadora y se ayuda al otro, insistiendo en que cree que debe hacer en relación a lo que puede realizar.

Para que el modelo empático se lleve a cabo correctamente debemos tener en cuenta unas fases que se deben seguir. La primera es la de identificación, en la que nos vemos reflejados con la persona y con la situación. La segunda es la fase de incorporación y repercusión, se trata de incorporar los elementos de la experiencia del paciente que son semejantes a los propios. Por último la fase de separación, muy importante para evitar el temido burn-out, se trata de apartarse de la implicación sentimental, manteniendo una distancia para evitar sentimientos de culpa (1).

\section{f) Habilidades y requisitos de conocimiento.}

Autoconocimiento, empatía, respeto, observación, escucha activa y conciencia de las propias habilidades, son los requisitos para poder formar a un profesional para poder realizar una correcta relación terapéutica. Por lo tanto son los objetivos que se deberían incluir en la actualidad en las escuelas de enfermería, y no como pasa actualmente, que estas 
habilidades se creen innatas. Pero cierto es que son habilidades y por lo tanto se han de poner en práctica para poder potenciarlas, si no es de esta manera ni siquiera sabremos si se está capacitado para entrenarlas (14). Actualmente los profesionales de enfermería no tienen una formación obligatoria en este aspecto, teniendo en cuenta, como hemos dicho anteriormente, que uno de los aspectos que los pacientes más en cuenta tienen es la comunicación que se establece con el profesional sanitario (15), si no sabemos comunicarnos y establecer una eficaz relación terapéutica, ¿cómo estaremos tratando al paciente? ¿Qué visión tendrá el paciente del equipo de enfermería? ¿Trataremos la globalidad de necesidades del paciente? Es importante tener en cuenta cómo y qué palabras utilizamos para empezar la conversación terapéutica ya que si son las adecuadas pueden ayudar a que se establezca más rápidamente una relación de confianza entre el paciente y la enfermera. Cada mirada, cada gesto comunica algo, y si utilizamos esto correctamente se podrá demostrar empatía e interés. Pero también esto es importante para ver la reacción del paciente y saber por sus gestos y miradas cómo se encuentra o lo que piensa (16).

\section{g) "Burn ouf" un enemigo de la enfermería.}

El hecho de no tener en cuenta este aspecto tan importante de la profesión enfermera puede llegar a desmotivar al profesional, hasta el punto de llegar a realizar "puras técnicas" sin tener en cuenta la globalidad de la "persona" a la que intentamos ayudar a conseguir un grado óptimo de salud. La "despersonalización" de la profesión junto a la falta de compañerismo, motivación y otros factores puede desembocar en el síndrome de burn out.

Si la relación terapéutica se establece después de haber adquirido conocimientos y habilidades, no quema sino que es vivificante. Se trata de no confundir relación de ayuda con amistad, empatía con simpatía, de no proyectar las propias preocupaciones en el paciente, de no hacer que el paciente dependa del profesional sino favorecer su autonomía, intentando que alcance el mayor bienestar. La relación es vivificante cuando el profesional conoce sus posibilidades y es capaz de poner en movimiento el potencial y recursos que tiene el paciente o la comunidad para hacer frente a su situación (7).

h) El arte de las habilidades sociales y la importancia de su entrenamiento en la formación de profesionales (presentación de estudios).

En un estudio realizado por Marín y León en dos grupos de alumnos de enfermería, se demostró a través del entrenamiento de habilidades sociales con un programa de entrenamiento de éstas, que fue eficaz incrementar las habilidades sociales de los sujetos expuestos en la intervención frente a los que no lo estaban. Concluyendo que el currículum académico de los profesionales de enfermería debería incluir procedimientos que capaciten al sujeto para establecer, mejorar y mantener relaciones interpersonales, con lo que evitaríamos el estrés al que se ve sometido el personal inexperto, además de mejorar su comunicación con el paciente y otros profesionales del equipo sanitario (4).

En otro estudio que se realizó en el Hospital Público de Fortaleza-CE en Portugal, basado en la teoría de Peplau y con el objetivo de analizar el proceso de comunicación terapéutica, participaron 14 pacientes y 12 enfermeros. Los resultados fueron que durante toda la estancia del paciente en el hospital había comunicación e interacción pero en algunas ocasiones no como debería ser. Y se concluyó que esto se debía a que la enfermera no hacía prioridad, en su tiempo de trabajo, a las visitas de los pacientes y a que los pacientes no diferenciaban a las enfermeras de otros componentes del equipo sanitario (17). 


\section{i) Cuidado recíproco enfermera/paciente}

La base fundamental de la relación terapéutica es el trabajo en equipo o "concordance" entre paciente y enfermera, y la identificación de objetivos entre ambos. Si la relación entre profesional sanitario y paciente no es efectiva, hay mas problemas y menos resultados. Actualmente muchos profesionales no son conscientes del potencial de curación de la relación terapéutica. Como afirma $M c D o n a l d ~ P$. una efectiva relación terapéutica es buena tanto para el paciente como para la enfermera, ya que el cuidado que se establece es recíproco (16). Al establecer esta relación es obvio que el profesional ayuda al paciente, pero el sanitario también recibe algo a cambio tanto en el ámbito profesional como personal (facilita el crecimiento personal y laboral) (7).

\section{CONCLUSIÓN}

Para conseguir el entendimiento global del ser particular que tenemos delante, es importante ayudar a la persona desde una concepción holística, esto requiere el establecimiento de una relación terapéutica enfermera-paciente, en la que, sin duda, el enriquecimiento va a ser mutuo. El buen profesional de enfermería tendrá que proponerse un conocimiento profundo de la persona con la que va a establecer la relación, habiendo adquirido previamente las habilidades y conocimientos necesarios para desarrollar una eficaz relación de ayuda (4).

La comunicación verbal es muy importante porque permite clarificar las ideas que el paciente intenta transmitirnos, sin embargo la traducción del lenguaje y expresión no verbal son igual de importantes. Es decir, el profesional sería capaz de traducir lo que sin decir nada, expresa la corporalidad del paciente en toda su complejidad (6).

El entrenamiento y aplicación de unas habilidades sociales sólidas tales como: autoconocimiento, observación, asertividad, empatía, respeto, observación, escucha activa, consciencia de las propias facultades y límites es fundamental para conseguir el bienestar del paciente. Estas son necesarias para establecer una comunicación efectiva con el paciente. La enfermera es el centro de referencia de la relación terapéutica, y a la que sin lugar a dudas el paciente se dirige en primera instancia cuando acontece algo importante para él o le preocupa algo y necesita hablarlo. Por lo tanto es importante adquirir estas habilidades y conocimientos a través de las escuelas de enfermería, así se irá dejando atrás el modelo paternalista y nos dirigiremos hacia una relación terapéutica basada en el diálogo abierto, interacción y la escucha activa (1). Como se dice en lenguaje coloquial nadie "nace con todo sabido" y hace ya tiempo que somos conscientes de las carencias en el sistema de formación de profesionales de enfermería (15). Si los estudiantes de enfermería están entrenados en estas habilidades, conseguiremos profesionales mejores preparados, que contemplarán a las personas en su globalidad, proporcionando una atención integral.

Si no se toman medidas, aparece el peligro inminente de entrar en la espiral sin salida del burn-out, en la que los profesionales que podrían sentirse realizados con su trabajo, se vuelven unos autómatas desconocidos incluso para sí mismos y acaban por olvidar completamente el sentido de la profesión (7). Podríamos decir que el burn-out es una desprofesionalización que causa muchos estragos, y que contrariamente a lo que piensa mucha gente no se debe a la relación terapéutica en sí, ni a las vivencias difíciles exclusivamente. Un profesional es capaz de ayudar al paciente conociendo sus posibilidades y limitaciones, sin proyectar sus preocupaciones en él, sino ayudándole a tomar conciencia de las armas que tiene para afrontar la situación. Así podemos decir que la enfermería es un arte y una ciencia. 
Para concluir, si enfermería no tiene las habilidades necesarias no podrá realizar una correcta alianza terapéutica con los pacientes. Por lo tanto es necesario e importante mantener y desarrollar estas habilidades en todos los profesionales de enfermería (8).

\section{REFERENCIAS BIBLIOGRÁFICAS}

1. Hurtado S. Enfermería y la relación de ayuda. Excelencia enfermera 2004,0: sin página.

2. García M. et al. Reflexiones en torno a la relación terapéutica. Índex Enferm (Gran) 2004; 47: 44-48.

3. Evans A. Transference in the nurse-patient relationship. Journal of psychiatric and mental health nursing 2007; 14: 189-195.

4. Marin M. et al. Entrenamiento en habilidades sociales. Psicothema 2001; 13 (2): 247-251.

5. García A. et al. Factores personales en la relación terapéutica. Revista de la asociación española de neuropsiquiatría 2005; 25 (96): 29-36.

6. Oliverira M. et al. Un ensayo sobre la comunicación en los cuidados de enfermería utilizando los sentidos. Enfermería global 2006; 8: 1.

7. Cibanal L. et al. La relación de ayuda es vivificante, no quema. Cultura de los cuidados; 2001: 5 (10): 88-99.

8. Brough C. Developing and maintaining a therapeutic relationship: part 1. Gerontological care and practice 2004, 16 (8).

9. Warelow P. et al. Care: what nurses say and what nurses do. Holistic nursing practice 2008 may-june; 146-153.

10. College of Nurses of Ontario. Therapeutic nurse-client relationship. The standard of care 2008 may; 3-17.

11. Stickley $\mathrm{T}$. et al. The art of listening in the therapeutic relationship. Mental helath practice 2006; 9 (5): 12-18.

12. Hernández M. Sociedad española de psicooncología. Psicooncología 2007; 4 (2-3): 523 526.

13. AIFICC, "Associació d'Infermeria Familiar i Comunitaria de Catalunya", Guia per l'escolta activa

$<$ http://www.aificc.cat/06_PUBLICACIONS/Guies\%20d'actuació/escolta\%20activa.pdf> [en

línea] última consulta: 15 de enero del 2009.

14. Foster. T. et al. The therapeutic relationship: dead or merely impeded by tecnology? British Journal of Nursing 2005; 14 (13): 698-702.

15. Mcqueen A. et al. Nurse patient relationship and partnership in hospital care. Journal of clinical nursing 2009; 9: 723-731.

16. McDonald P. Developing a therapeutic relationship. Practice nurse 2003; 26 (6): 56-61.

17. Carvalho A. et al. Comunicagao terapéutica em enfermagem: instrumento essencial do cuidado. Revista Brasileira de Enfermagem 2008; 61(3): 312-318.

18. O'Brien A. The therapeutic relationship: historical development and contemporary signifcance. Journal of Psychiatric and Mental Health Nursing 2001; 8: 129-137.

19. MCallister $M$. et al. Conversation starters: re-examining and reconstructing first encounters within the therapeutic relationship. Journal of Psychiatric and Mental Health Nursing 2004; 11: 575-582.

20. Moyle W. Nurse-patient relationship: a dichtomy of expectations. International Journal of Mental Health Nursing 2003; 12: 103-109.

21. Rodríguez J. Comunicación entre el profesional sanitario y el paciente. Universidad de Antioquia 2004: sin página. 
ISSN 1695-6141 